\title{
Controlled excitations of surface plasmons via optical soliton photons in the quantum regime
}

\author{
Evren Karakaya, Kaan Güven and Özgür E Müstecaplıoğlu \\ Department of Physics, Koç University, İstanbul 34450, Turkey \\ E-mail: ekarakaya@ku.edu.tr, kguven@ku.edu.tr and omustecap@ku.edu.tr
}

Received 15 September 2013

Accepted for publication 18 November 2013

Published xxx

\begin{abstract}
We examine quantum dynamics of surface plasmons weakly coupled to optical soliton photons in a metal-dielectric-nonlinear Kerr medium interface. We consider a photon-number-dependent coupling model which is a heuristic extension of the classical soliton excitation of surface plasmons in the quantum limit. The coupling coefficient additionally depends on the distance between the dielectric and the metal. We propose a practical scheme to control the number of surface plasmons by a Gaussian variation of the metal-dielectric spatial separation. Our numerical simulations show that the curvature of the Gaussian distance profile can be used for controlling the number of surface plasmons.
\end{abstract}

Keywords: $\mathrm{xxx}$

(Some figures may appear in colour only in the online journal)

\section{Introduction}

Coupling surface plasmons (SPs) [1] to optical solitons in nonlinear (Kerr-type) dielectric waveguides has been proposed as an efficient way to control them [2]. This system offers periodical soliton-SP conversion, nonadiabatic switching and hybridized soliton-plasmon bound states.

The resonant interaction coefficient between the SP and the soliton depends on the intensity of the soliton [2]. This leads to rich dynamical features as have been discussed in detail in the literature [3-5]. A phase space analysis classifies the model system as a new type of Josephson junction [3]. Recently, the dissipative effect has been taken into account to introduce a more reliable representation of the practical system [4], where it has been found that robust dynamical phases can be achieved despite the presence of the dissipation by expansion of the Hopf bifurcations to stable limit cycles. Hybridized soliton-plasmon bound states, or the so-called soliplasmons, arise as eigenmodes of the nonlinear Maxwell's equations for the metal-dielectric-Kerr medium (MDK) interface [5].

All these appealing dynamical features however can be questionable in the limit of low soliton and SP amplitudes due to the possible influence of quantum corrections in such a weak excitation limit. This work aims to investigate the quantum dynamics of the system in the weak excitation limit. In particular, our objective is to explore controlled population transfer from soliton photons to SPs.

The paper is organized as follows. In section 2, we present our model system and the equations of motion. Our control method is described here as well. Section 3 reports our results obtained by solving the model equations. We conclude in section 4 .

\section{The model system}

We consider the system of a nonlinear dielectric waveguide separated by a linear dielectric layer from a metal [2]. The evanescent tail of the soliton in the waveguide excites SPs on the metal surface. The soliton-plasmon coupling depends on the soliton amplitude. The nonlinear Kerr medium is assumed to be at a distance $d$ from the metal so that small overlap of the SP and soliton in the linear dielectric layer validates weak coupling and linear SP treatments.

The strong [2,6] and weak [6] soliton amplitude $\left|C_{\mathrm{s}}\right|$ behaviors of the coupling coefficient $q$ can be described by a fitting function [4]

$$
q\left(\left|C_{\mathrm{s}}\right|\right) \simeq\left|C_{\mathrm{s}}\right| \mathrm{e}^{-\sigma k d \sqrt{\gamma / 2}\left|C_{\mathrm{s}}\right|}
$$


Here $k$ is the wavenumber in the dielectric, $\gamma$ is the nonlinearity parameter and $\sigma$ is a material-dependent constant. We consider the case of weak soliton amplitude for which $q \sim\left|C_{\mathrm{s}}\right|$ and assume the special case of symmetric soliton-plasmon interaction [6].

The essential physics of the system is described by intensity-dependent coupling of nonlinear and linear oscillators. As the quantum mechanical counterpart that captures this physics for weak soliton and plasmon excitations we consider a model Hamiltonian

$$
H=\omega_{\mathrm{p}} \hat{a}_{\mathrm{p}}^{\dagger} \hat{a}_{\mathrm{p}},+\omega_{\mathrm{s}} \hat{a}_{\mathrm{s}}^{\dagger} \hat{a}_{\mathrm{s}}+u \hat{a}_{\mathrm{s}}^{\dagger} \hat{a}_{\mathrm{s}}^{\dagger} \hat{a}_{\mathrm{s}} \hat{a}_{\mathrm{s}}+g(t)\left(f\left(\hat{n}_{\mathrm{s}}\right) \hat{a}_{\mathrm{s}}^{\dagger} \hat{a}_{\mathrm{p}}+\text { h.c. }\right)
$$

Here, the annihilation and creation operators $\hat{a}_{\alpha}$ and $\hat{a}_{\alpha}^{\dagger}$, respectively, are for the soliton $(\alpha=\mathrm{s})$ photons and for the SPs $(\alpha=\mathrm{p})$. They obey the Weyl-Heisenberg algebra of the bosonic fields with the frequencies $\omega_{\mathrm{s}}$ and $\omega_{\mathrm{p}}$. We denote the nonlinearity parameter by $u$. We allow the coupling coefficient to be time dependent for the purpose of controlling the population dynamics. Nonlinearity of the soliton-plasmon coupling is described by an operator function $f\left(\hat{n}_{\mathrm{S}}\right)=\sqrt{\hat{n}_{\mathrm{s}}}$ with $\hat{n}_{\mathrm{s}}=\hat{a}_{\mathrm{s}}^{\dagger} \hat{a}_{\mathrm{s}}$ being the number operator of the soliton photons. This form is taken to represent the case of weak soliton amplitude.

The Heisenberg equations of motion for $H$ are found to be

$$
\begin{aligned}
& \mathrm{i} \frac{\mathrm{d} \hat{a}_{\mathrm{s}}}{\mathrm{d} t}=\omega_{\mathrm{s}} \hat{a}_{\mathrm{s}}+2 u \hat{a}_{\mathrm{s}}^{\dagger} \hat{a}_{\mathrm{s}}^{2}+g(t)\left(f\left(\hat{n}_{\mathrm{s}}\right) \hat{a}_{\mathrm{p}}\right. \\
& \left.+\Delta\left(\hat{n}_{\mathrm{s}}\right) \hat{a}_{\mathrm{s}} \hat{a}_{\mathrm{s}}^{\dagger} \hat{a}_{\mathrm{p}}+\hat{a}_{\mathrm{p}}^{\dagger} \hat{a}_{\mathrm{s}} \Delta\left(\hat{n}_{\mathrm{s}}\right) \hat{a}_{\mathrm{s}}\right), \\
& \mathrm{i} \frac{\mathrm{d} \hat{a}_{\mathrm{p}}}{\mathrm{d} t}=\omega_{\mathrm{p}} \hat{a}_{\mathrm{p}}+g(t) \hat{a}_{\mathrm{s}} f\left(\hat{n}_{\mathrm{s}}\right)
\end{aligned}
$$

where we define $\Delta\left(\hat{n}_{\mathrm{s}}\right)=f\left(\hat{n}_{\mathrm{s}}+\hat{1}\right)-f\left(\hat{n}_{\mathrm{s}}\right)$ with $\hat{1}$ being the unit operator. In the classical limit we replace the operators with the $c$-numbers such that $\hat{a}_{\alpha} \rightarrow a_{\alpha}$ and take $\Delta\left(n_{\mathrm{s}}\right) \rightarrow 0$. In a frame rotating at $\omega_{\mathrm{s}}$ we have $a_{\mathrm{s}, \mathrm{p}}=C_{\mathrm{s}, \mathrm{p}} \exp \left(\mathrm{i} \omega_{\mathrm{s}} t\right)$. The equations of motion then become

$$
\begin{aligned}
& \mathrm{i} \frac{\mathrm{d} C_{\mathrm{s}}}{\mathrm{d} t}=2 u\left|C_{\mathrm{s}}\right|^{2}\left|C_{\mathrm{s}}\right|+g(t) f\left(\left|C_{\mathrm{s}}\right|\right) C_{\mathrm{p}}, \\
& \mathrm{i} \frac{\mathrm{d} C_{\mathrm{p}}}{\mathrm{d} t}=\left(\omega_{\mathrm{p}}-\omega_{\mathrm{s}}\right) C_{\mathrm{p}}+g(t) f\left(\left|C_{\mathrm{s}}\right|\right) C_{\mathrm{s}},
\end{aligned}
$$

where in the classical limit the soliton photon number is large and hence we should use $f\left(\left|C_{\mathrm{s}}\right|\right)=\exp \left(-\sigma k d \sqrt{\gamma / 2}\left|C_{\mathrm{s}}\right|\right)=$ $q\left(\left|C_{\mathrm{s}}\right|\right)$. Identifying $\omega_{\mathrm{s}}-\omega_{\mathrm{p}}=v_{\mathrm{p}}, 2 u=-\gamma / 4$ and $g(t)=$ $1 / 2$ we see that our quantum model equations reduce to the classical ones in [2]. Our time variable $t$ corresponds to the spatial variable $\zeta=k z$ with $z$ being the coordinate along the propagation axis. Such resemblance of space and time variables [7, 8] and canonical quantization of field variables $[9,10]$ are standard techniques for quantum treatment of propagating fields. These identifications suggest that the quantum model parameters should be considered as effective and dimensionless.

Negative $u$ values correspond to attractive interactions among photons to compensate the dispersive free evolution to yield soliton propagation, and the form of the nonlinear

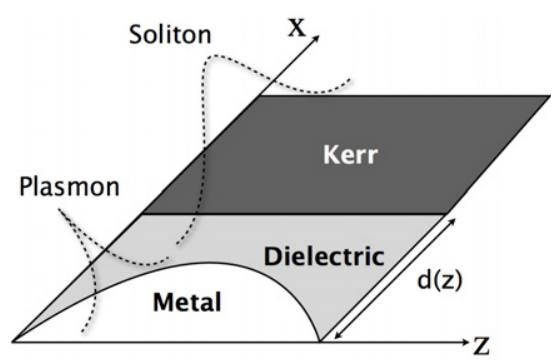

Figure 1. Schematic representation of the MDK system, as in [2], consists of a Kerr-type nonlinear waveguide and a metal separated from each other by a linear dielectric layer. We assume that the thickness $d(z)$ of the dielectric layer varies along the propagation axis $z$. Dashed curves indicate soliton and SP transverse profiles.

The soliton tail leaking out of the Kerr medium induces SP excitations by evanescent coupling along the $x$-axis in the dielectric. The system is considered as uniform along the $y$ direction.

interaction is the same as the standard quantum models of single mode soliton propagation [11, 12]. Quantum models of SPs [13] and their single-photon excitations are described by similar Hamiltonians as $H$ [14]. The new elements in our model are the nonlinearity of the photonic subsystem and the nonlinearity of the soliton-plasmon coupling at the quantum level. Bound states of the classical model are defined as soliplasmons which occur at an intensity-dependent resonance $\omega_{\mathrm{p}}=\omega_{\mathrm{s}}+2 u\left|C_{\mathrm{s}}\right|^{2}[2,5,6]$, which can be obtained with our quantum model in the classical limit. To search for the quantum nature of soliplasmons one needs to employ the so-called $f$-deformed algebra due to the nonlinear coupling [15]. Here we assume their existence in the quantum model and consider the quantum dynamics in the vicinity of soliplasmon resonance as their classical counterparts [2].

We propose that the time-dependent coupling can be implemented by varying the spatial distance between the metal and the waveguide as shown schematically in figure 1 . For a concrete and simple model we assume that $g(d) \sim 1 / d$ for the weak soliton amplitude. If $d(z)$ varies in a Gaussian form along the $z$-axis then such a spatial variation of the material curvature is translated into a Gaussian temporal profile for the interaction coefficient such that

$$
g(t)=g \exp \left(-\Lambda t^{2}\right)
$$

This is due to the resemblance of the spatial variable $\zeta=$ $k z$ in the classical model to the time variable $t$ in the quantum model. Here the control parameter $\Lambda$ is assumed to be adjusted by the curvatures of the metal or the dielectric waveguide. The essential requirements concerning the weak coupling approximation are still fulfilled under such a distance variation provided that the closest distance in the Gaussian profile between the metal and the dielectric is sufficiently wide enough for the small overlap of the plasmon and soliton fields. The material curvature or the width of the Gaussian is the basic control parameter in our population transfer scheme. In the next section, we report our results for the typical numerical simulations to discuss the success of the proposed control method. 

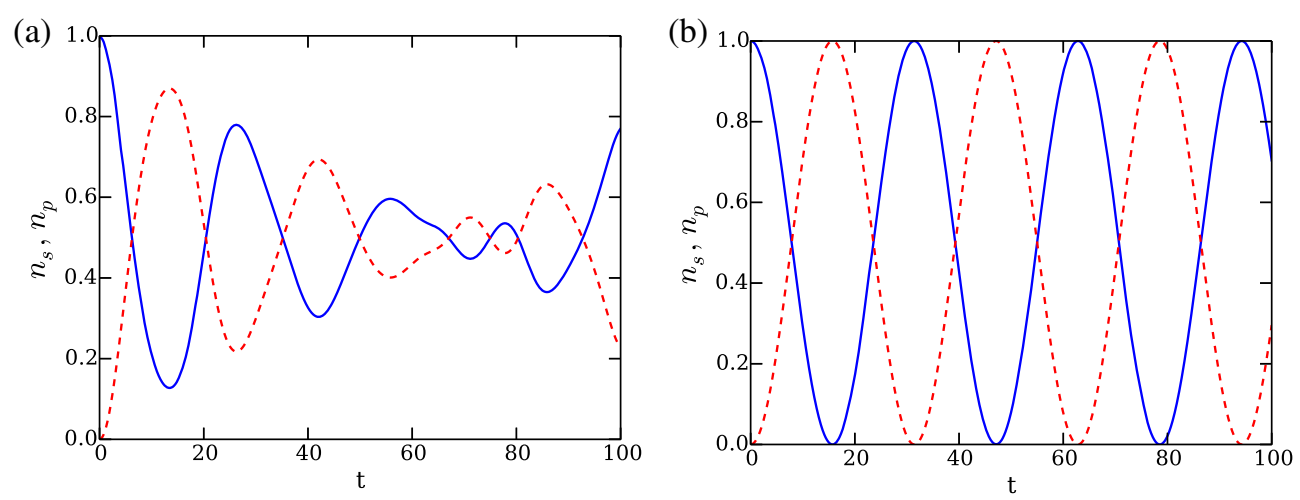

Figure 2. Time $(t)$ dependence of the soliton photon and SP number populations ( $n_{\mathrm{s}}$ and $n_{\mathrm{p}}$, respectively) in the case of time-independent coupling $g(t)=g$. Initially, the plasmon is in the vacuum state while the photon is in (a) coherent state $|\alpha=1\rangle$ and in (b) Fock state $\left|n_{\mathrm{s}}=1\right\rangle$. In both cases the system is lossless with $\kappa=0$, in the weak coupling regime $g=0.1 \omega_{\mathrm{p}}$, and the nonlinearity coefficient is $u=-0.01 \omega_{\mathrm{p}}$. Time is dimensionless and scaled by the plasmon frequency $\omega_{\mathrm{p}}$. In the strong coupling similar curves are found but with more rapid oscillations.
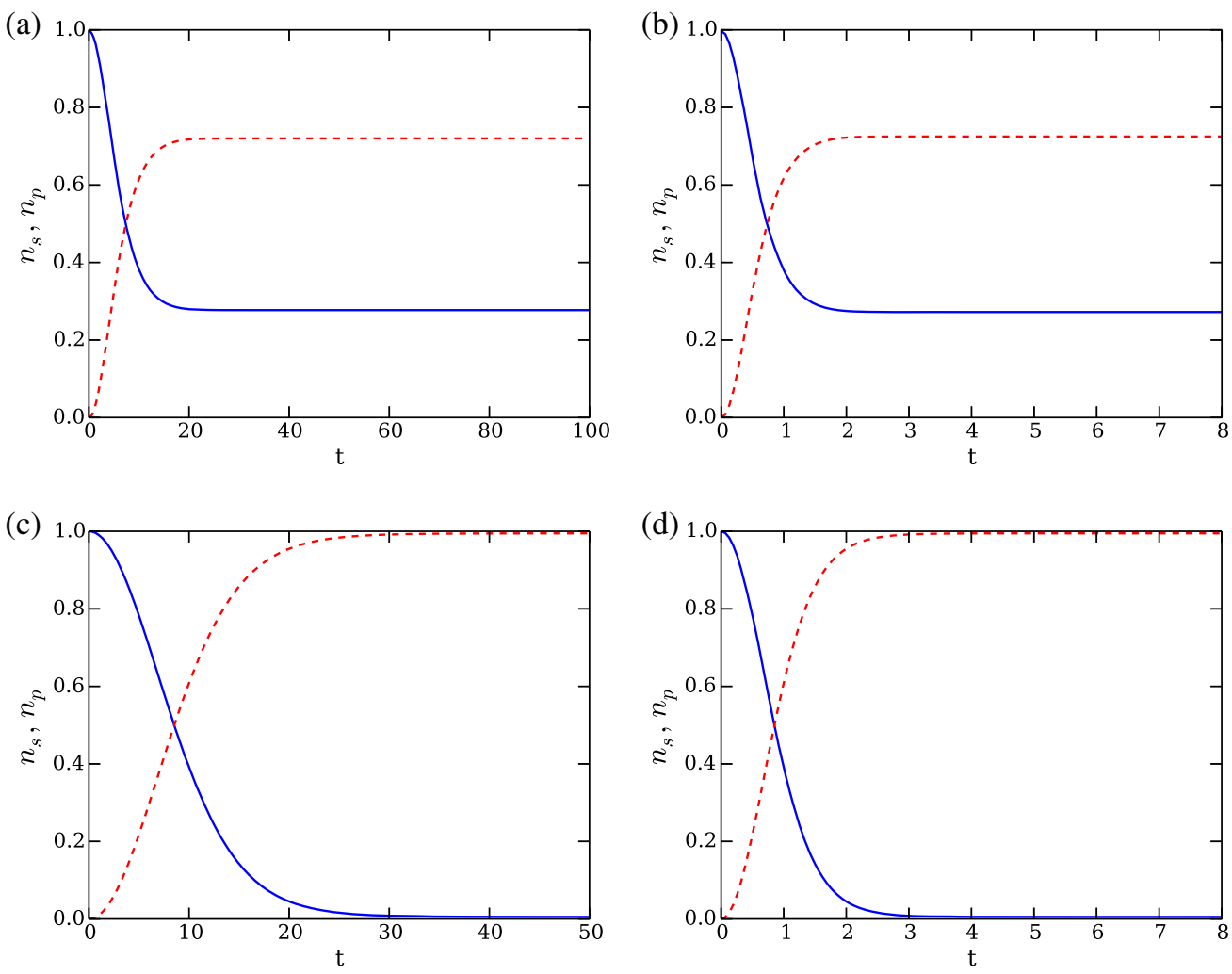

Figure 3. Time $(t)$ dependence of the soliton photon and SP number populations ( $n_{\mathrm{s}}$ and $n_{\mathrm{p}}$, respectively) in the case of time-dependent coupling $g(t)$. Initially, the plasmon is in the vacuum state. In all cases the nonlinearity coefficient is $u=-0.01 \omega_{\mathrm{p}}$. The interaction coefficient has the Gaussian time dependence and its width parameter $\Lambda$ and its amplitude $g$ are varied in the subfigures as (a) $g=0.1 \omega_{\mathrm{p}}$, $\Lambda=0.01$ and (b) $g=\omega_{\mathrm{p}}, \Lambda=1$ for the soliton initially in the coherent state $|\alpha=1\rangle$; and (c) $g=0.1 \omega_{\mathrm{p}}, \Lambda=0.0035$ and (d) $g=\omega_{\mathrm{p}}$, $\Lambda=0.35$ for the soliton in the $\left|n_{\mathrm{s}}=1\right\rangle$ Fock state. Time is dimensionless and scaled by the plasmon frequency $\omega_{\mathrm{p}}$.

\section{Results and discussions}

We solve the quantum optical master equation

$$
\mathrm{i} \frac{\mathrm{d} \hat{\rho}}{\mathrm{d} t}=[\hat{H}, \hat{\rho}]+\frac{\kappa}{2}\left(\hat{a}_{\mathrm{p}} \hat{\rho} \hat{a}_{\mathrm{p}}^{\dagger}-\hat{a}_{\mathrm{p}}^{\dagger} \hat{a}_{\mathrm{p}} \hat{\rho}-\hat{\rho} \hat{a}_{\mathrm{p}}^{\dagger} \hat{a}_{\mathrm{p}}\right)
$$

under Born-Markov approximations and for a particular preparation of the system. We specifically consider that the soliton photon is either in a single-photon Fock number state $\left|n_{\mathrm{s}}=1\right\rangle$ or in coherent state $|\alpha=1\rangle$ initially, while the plasmon is in vacuum state $\left|n_{\mathrm{p}}=0\right\rangle$. We use QuTiP (Quantum
Toolbox in Python) programming language [16, 17] to numerically integrate the master equation. This open source software uses the matrix representation of the operators and employs finite differencing in time to evolve the initial density matrix operator $\hat{\rho}(t)$. We take five-dimensional Fock states as the Hilbert spaces of the soliton and plasmon subsystems. We verified the convergence of our results by considering larger Hilbert spaces. We determine the population dynamics of the soliton photons and the SPs by evaluating $n_{\mathrm{s}}=\operatorname{Tr}\left(\hat{\rho}(t) \hat{a}_{\mathrm{s}}^{\dagger} a_{\mathrm{s}}\right)$ and $n_{\mathrm{p}}=\operatorname{Tr}\left(\hat{\rho}(t) \hat{a}_{\mathrm{p}}^{\dagger} a_{\mathrm{p}}\right)$, respectively. 
We assumed that the metallic loss of plasmons is the only decay channel of the system. Our simulations reveal a simple qualitative change from the lossless behaviors when we introduce a finite phenomenological loss rate for the metallic subsystem $\kappa$. Instead of staying robustly at a constant population after the excitation by the soliton, SPs decay due to the metallic loss and hence their population dynamics appears as a pulse in time. In our reported results below we shall only present the case of $\kappa=0$ to emphasize the essential physical mechanism of population transfer. In practice, it should be understood that SPs would decay after the transfer at a rate determined by $\kappa$.

We use the scaled dimensionless time $t^{\prime}=\omega_{\mathrm{p}} t$. For the brevity of notation, we shall drop the prime and refer $t$ as the dimensionless scaled time. We scale $u, g$ and $\omega_{\mathrm{s}, \mathrm{p}}$ by $\omega_{\mathrm{p}}$ as well. We take $\omega_{\mathrm{s}}=\omega_{\mathrm{p}}$ and small nonlinearity $u=0.01 \omega_{\mathrm{p}}$ to remain in the vicinity of the soliplasmon resonance. We consider the cases of weak and strong coupling by $g=0.1 \omega_{\mathrm{p}}$ and $g=\omega_{\mathrm{p}}$, respectively. For simplicity we suppose that the heuristic model Hamiltonian does not change in the case of strong coupling.

In order to develop efficient control, we first examine the dynamics of the number populations of the soliton photons and the SPs for a constant coupling $g(t)=g$. Our typical results are given in figure 2. In the left panel, figure 2(a), we show the time dependence of the populations for the initially coherent preparation of the soliton state. The dynamics is irregular due to the contribution of the higher number of photon states. As the interaction is highly nonlinear, in contrast to the linear interaction, we do not see perfect revivals but incomplete and irregular oscillations after the initial collapse of the population. This behavior suggests that we should not expect complete population transfer in this case. Figure 2(b) shows the case of Fock state initial preparation. In contrast to the coherent preparation, a profound quantum soliton prepared in a single-photon Fock state allows for complete and regular population transfer. This can be exploited then by the Gaussian approach to turn on and turn off the interaction with a proper timing for a complete and robust population transfer.

Applying the Gaussian temporal profile, we get the typical results illustrated in figure 3 . For the coherent state we find rather partial success in transferring the soliton population to the plasmon subsystem. For the weak coupling case, a small width parameter is sufficient to transfer $\sim 70 \%$ of soliton photons to SPs as shown in figure 3(a). This can be achieved in the strong coupling case, plotted in figure $3(\mathrm{~b})$, as well by using a larger width parameter $\Lambda=$ 1. On the other hand, when the soliton is prepared in the Fock state $\left|n_{\mathrm{s}}=1\right\rangle$, complete population transfer can be obtained both in the weak coupling case and in the strong coupling case, plotted in figures 3(c) and in (d), respectively. Al-though here we demonstrated the case of complete transfer, a complete range of transfer ratios can be obtained by tuning the width parameter $\Lambda$. The interaction amplitude $g$ controls the time of transfer. Earlier transfer occurs at larger interaction amplitude $g$.

\section{Conclusions}

We examined a MDK interface where a soliton in the Kerr medium excites SPs on the metal in the quantum regime for the purpose of controlled population transfer from the soliton to the plasmon subsystem. We considered initially coherent and Fock state preparations of the soliton photons. We find that quantum dynamics of populations exhibit irregular revivals after the collapse time in the case of coherent preparation while regular coherent oscillations arise for the Fock number state preparation. This observation is then exploited by suggesting a simple control mechanism where the spatial separation between the waveguide and the metal is translated into a Gaussian time-dependent coupling coefficient. We find that complete and robust population transfer is possible for the Fock state preparation. The coherent case however yields only a partial success of transfer. The transfer time and the ratio can be controlled by the amplitude of the interaction and the width of the Gaussian, respectively. Our results can contribute to the efficient control over plasmon dynamics and excitations in the quantum regime.

\section{Acknowledgment}

We acknowledge financial support from TÜBİTAK (grant no. 111T285).

\section{References}

[1] Maier S A 2007 Plasmonics Fundamentals and Applications (New York: Springer)

[2] Bliokh K Y, Bliokh Y P and Ferrando A 2009 Phys. Rev. A 79041803

[3] Ekşioğlu Y, Müstecaplığlu Ö E and Güven K 2011 Phys. Rev. A 84033805

[4] Ekşioğlu Y, Müstecaplıoğlu Ö E and Güven K 2013 Phys. Rev. A 87023823

[5] Milián C, Ceballos-Herrera D E, Skryabin D V and Ferrando A 2012 Opt. Lett. 37 4221-3

[6] Ferrando A, Milián C and Skryabin D V 2013 J. Opt. Soc. Am. B 30 2507-22

[7] Shen Y R 1967 Phys. Rev. 155 921-31

[8] She C Y 1968 Phys. Rev. 176 461-7

[9] Yurke B, Grangier P, Slusher R E and Potasek M J 1987 Phys. Rev. A 35 3586-9

[10] Potasek M J and Yurke B 1987 Phys. Rev. A 35 3974-7

[11] Haus H A, Watanabe K and Yamamoto Y 1989 J. Opt. Soc. Am. B $61138-48$

[12] Tanaś R and Kielich S 1990 J. Mod. Opt. 37 1935-45

[13] González-Tudela A, Huidobro P A, Martín-Moreno L, Tejedor C and García-Vidal F J 2013 Phys. Rev. Lett. 110126801

[14] Tame M S, Lee C, Lee J, Ballester D, Paternostro M, Zayats A V and Kim M S 2008 Phys. Rev. Lett. 101190504

[15] Man'ko V I, Marmo G, Sudarshan E C G and Zaccaria F 1997 Phys. Scr. 55528

[16] Johansson J R, Nation P D and Nori F 2012 Comput. Phys. Commun. 183 1760-72

[17] Johansson J, Nation P and Nori F 2013 Comput. Phys. Commun. 184 1234-40 


\section{QUERY FORM}

JOURNAL: ps

Author: E Karakaya et al

Title: Controlled excitations of surface plasmons via optical soliton photons in the quantum regime

ARTICLE ID: pstop489651

\section{Page 1}

Q1.

Author: Please supply a minimum of three (and a maximum of seven) keywords relevant to your article.

\section{Page 4}

Q2.

Author: Please check the details for any journal references that do not have a blue link as they may contain some incorrect information. Pale purple links are used for references to arXiv e-prints.

Author: Please be aware that the colour figures in this article will only appear in colour in the Web version. If you require colour in the printed journal and have not previously arranged it, please contact the Production Editor now. 